\title{
Knockdown of Indian hedgehog protein induces an inhibition of cell growth and differentiation in osteoblast MC3T3-E1 cells
}

\author{
ANG DENG ${ }^{1}$, HONGQI ZHANG ${ }^{1}$, MINYU HU $^{2}$, SHAOHUA LIU ${ }^{1}$, \\ QILE GAO $^{1}$, YUXIANG WANG ${ }^{1}$ and CHAOFENG GUO ${ }^{1}$ \\ ${ }^{1}$ Department of Spine Surgery, Xiangya Hospital of Central South University, Changsha, Hunan 410008; ${ }^{2}$ Department of \\ Nutrition and Food Hygiene, School of Public Health, Central South University, Changsha, Hunan 410078, P.R. China
}

Received December 27, 2016; Accepted July 20, 2017

DOI: $10.3892 / \mathrm{mmr} .2017 .7669$

\begin{abstract}
Indian hedgehog protein (Ihh) is evolutionarily conserved and serves important roles in controlling the differentiation of progenitor cells into osteoblasts. Ihh null mutant mice exhibit a failure of osteoblast development in endochondral bone. Although studies have demonstrated that Ihh signaling is a potent local factor that regulates osteoblast differentiation, the specific transcription factors that determine osteoblast differentiation remain unclear. Further studies are required to determine the precise mechanism through which Ihh regulates osteoblast differentiation. In the present study, Ihh was knocked down in osteoblast MC3T3-E1 cells using short hairpin RNA, to investigate the function of Ihh in osteoblast proliferation and differentiation and to examine the potential mechanism through which Ihh induces osteoblast apoptosis and cell cycle arrest. It was observed that the knockdown of Ihh induced a marked inhibition of cell growth and increased the apoptosis rate compared with the negative control osteoblasts. Downregulation of Ihh resulted in a cell cycle arrest at the G1 to S phase boundary in osteoblasts. In addition, the knockdown of Ihh decreased the alkaline phosphatase activity and mineral deposition of osteoblasts. The inhibitory roles of Ihh downregulation in osteoblast growth and differentiation may be associated with the transforming growth factor- $\beta /$ mothers against decapentaplegic homolog and tumor necrosis factor receptor superfamily member 11B/tumor necrosis factor ligand superfamily member 11 signaling pathways. Manipulating either Ihh expression or its signaling components may be of benefit for the treatment of skeletal diseases.
\end{abstract}

Correspondence to: $\operatorname{Dr}$ Shaohua Liu, Department of Spine Surgery, Xiangya Hospital of Central South University, 87 Xiangya Road, Changsha, Hunan 410008, P.R. China

E-mail: liushaohua_1@163.com

Key words: Indian hedgehog protein, osteoblast, differentiation, bone formation, mothers against decapentaplegic homolog, tumor necrosis factor receptor superfamily member $11 \mathrm{~B}$

\section{Introduction}

The hedgehog (Hh) genes, including Sonic hedgehog (Shh), Indian hedgehog (Ihh) and Desert hedgehog are evolutionarily conserved and serve important roles in controlling the differentiation of progenitor cells into either osteoblasts or chondrocytes (1). Dysfunction of Hh signaling leads to disruption of bone development and homeostasis (2).

Osteoblasts originate from pluripotent mesenchymal stem cells. The most important function of osteoblasts is to form mineralized bones. Osteoblasts express various phenotypic markers, including high alkaline phosphatase (ALP) activity, and synthesize collagenous and noncollagenous bone matrix proteins, including osteocalcin (OCN) (3). Osteoblasts express receptors for various hormones, including parathyroid hormone-related protein (PTHrP), runt-related transcription factor 2 (RUNX2) and tumor necrosis factor ligand superfamily member 11 (RANKL), which are involved in the regulation of osteoblast differentiation $(4,5)$.

It has been suggested that Ihh may induce adjacent perichondrial cells to differentiate into bone-forming osteoblasts (6). Ihh null mutant mice exhibited a failure of osteoblast development in endochondral bones (7). Ihh overexpression increased ALP activity and the level of OCN mRNA expression in MC3T3-E1 cells (8). In addition, recombinant Shh synergistically stimulated bone morphogenetic protein-induced ALP activity and the expression level of OCN mRNA in C3H10T1/2 cells (9). Although various studies have demonstrated that $\mathrm{Hh}$ signaling is a potent local factor that regulates osteoblast differentiation, the specific transcription factors that determine osteoblast differentiation remain unclear. Further studies are required to determine the precise mechanism through which Ihh regulates osteoblast differentiation.

In the present study, Ihh was knocked down in osteoblast MC3T3-E1 cells using short hairpin (sh)RNA, in order to investigate the function of Ihh in osteoblast proliferation and differentiation and to examine the potential mechanism through which Ihh may induce osteoblast apoptosis and cell cycle arrest.

\section{Materials and methods}

Cell line and cell transfection. The mouse osteoblast MC3T3-E1 cells were purchased from the American Type 
Culture Collection (Manassas, VA, USA). Cells were cultured routinely in Dulbecco's modified Eagle's medium/F-12 (Invitrogen; Thermo Fisher Scientific, Inc., Waltham, MA, USA), supplemented with $10 \%$ fetal bovine serum (Gibco; Thermo Fisher Scientific, Inc.), and cultured in a $37^{\circ} \mathrm{C}$ humidified atmosphere with $5 \% \mathrm{CO}_{2}$.

Knockdown of Ihh in cells was achieved by infection with a lentivirus containing Ihh shRNA sequences (Ihh-shRNA; Shanghai GenePharma Co., Ltd., Shanghai, China). Cells transfected with empty lentivirus were used as the negative control (NC), and untreated cells were used as the blank control. Cells $\left(5 \times 10^{5}\right.$ cells $\left./ \mathrm{ml}\right)$ were plated in 6 -well clusters $(1 \mathrm{ml})$ or 96 -well plates $(0.2 \mathrm{ml})$ and transfected with lentivirus $\left(10^{8} \mathrm{U} / \mathrm{ml}\right.$ ) for $48 \mathrm{~h}$ by using Lipofectamine 3000 (Thermo Fisher Scientific, Inc.). Transfected cells were used in further assays or RNA/protein extraction.

$R N A$ extraction and SYBR green reverse transcription-quantitative polymerase chain reaction (RT-qPCR) analysis. Total RNA was extracted from cells using TRIzol reagent (Invitrogen; Thermo Fisher Scientific, Inc.). RevertAid First Strand cDNA Synthesis kit (Thermo Fisher Scientific, Inc.) was used to reverse transcribe the mRNA to cDNA according to the manufacturer's protocol. The mRNA expression of Ihh, PTHrP, transforming growth factor (TGF) $-\beta$, OCN, RUNX2, tumor necrosis factor receptor superfamily member 11B (OPG), mothers against decapentaplegic homolog $(\mathrm{Smad}) 2$, Smad3, collagen $\alpha-1$ (X) chain (COL10A) and RANKL mRNA expression was measured using a SYBR Green qPCR assay (Takara Biotechnology Co., Ltd., Dalian, China) under the following conditions: $95^{\circ} \mathrm{C}$ for $5 \mathrm{~min}$, followed by 40 cycles of $95^{\circ} \mathrm{C}$ for $15 \mathrm{sec}$ and $60^{\circ} \mathrm{C}$ for $30 \mathrm{sec}$. The primers used in this study were as follows: Ihh, forward: 5'-CTCAGC CTGCTCTCACTACG-3', reverse: 5'-AAGCACATCCAA CCCACCTC-3'; PTHrP, forward: 5'-CGAGGTTCAAAG GTTTGCCTC-3', reverse: 5'-GGCCAGAGAAGCCTGTTA CC-3'; TGF- $\beta$, forward: 5 '-AGGGCTACCATGCCAACT TC-3', reverse: 5'-TGACACAGAGATCCGCAGTC-3'; OCN, forward: 5'-TCCTTTGGGGTTTGGCCTAC-3', reverse: 5'-CTTGGACACAAAGGCTGCAC-3'; RUNX2, forward: 5'-CGCCTCACAAACAACCACAG-3', reverse: 5'-TCACTG TGCTGAAGAGGCTG-3'; OPG, forward: 5'-CTGGAACCC CAGAGCGAAAT-3', reverse: 5'-GCGTTTACTTTGGTG CCAGG-3'; Smad2, forward: 5'-GTTCCTTTCCTCCTC CGCTC-3', reverse: 5'-AGTCTCTTCACAACTGGCGG-3'; Smad3, forward: 5'-TTCACTGGTGCTGGGGTTAG-3', reverse: 5'-GGTAGGGATTCACGCAGACC-3'; COL10A, forward: 5'-CTCCCAGCACGCAGAATC-3', reverse: 5'-TTC CCTACAGCTGATGGTCC-3'; RANKL, forward: 5'-GGA GTTGGCCGCAGACAAGA-3', reverse: 5'-TGATGTGCT GTGATCCAACGA-3' and $\beta$-actin, forward: 5'-GCAGGA GTATGACGAGTCCG-3', reverse: 5'-AACAACGCATCT CATATTTGGAA- 3 '. The expression of $\beta$-actin was used as an endogenous control. Data were processed using the $2^{-\Delta \Delta C q}$ method (10).

Cell Counting Kit-8 (CCK-8) cell proliferation assay. Cell proliferation rates were measured using a CCK- 8 assay (Beyotime Institute of Biotechnology, Haimen, China). A total of $0.5 \times 10^{4}$ cells/well were seeded into a 96 -well plate for $24 \mathrm{~h}$, transfected with the indicated lentiviruses, and further incubated for 24,48 and $72 \mathrm{~h}$, respectively. A total of $10 \mu \mathrm{l} \mathrm{CCK}-8$ reagents were added to each well at $1 \mathrm{~h}$ prior to the endpoint of incubation. The optical density at $490 \mathrm{~nm}$ for each well was determined using a microplate reader.

Flow cytometric analysis of apoptosis with Annexin-V/PI double staining. An annexin $\mathrm{V}$ apoptosis detection kit (Thermo Fisher Scientific, Inc.) was used for the analysis of apoptosis. Following transfection, the cells were trypsinized, harvested and resuspended. A total of $2 \times 10^{5}$ cells were harvested and washed twice with cold PBS, and subsequently resuspended in $500 \mu \mathrm{l}$ binding buffer. A total of $10 \mu \mathrm{l}$ annexin V-fluorescein isothiocyanate and $10 \mu \mathrm{l}$ propidium iodide was added to the solution and mixed well. Following 15 min of incubation, the cells were analyzed by flow cytometric analysis (BD Biosciences, San Jose, CA, USA) with FCS Express 6 flow cytometry software (FCS Express 6.04.0015, De Novo Software, Los Angeles, CA, USA).

Cell cycle analysis. Cell cycle analysis was performed using flow cytometry. A total of $1 \times 10^{4}$ cells were seeded into each well of a 6-well plate. Following $48 \mathrm{~h}$ of transfection with Ihh-shRNA or empty lentivirus, the cells were harvested and fixed in $70 \%$ ice-cold ethanol for $24 \mathrm{~h}$, followed by staining with propidium iodide at $37^{\circ} \mathrm{C}$ for $15 \mathrm{~min}$. The different phases of the cell cycle were analyzed using a FACSCalibur instrument (BD Biosciences) FCS Express 6 software.

ALP assay. An ALP Assay kit (Beyotime Institute of Biotechnology) was used to detect ALP activity, according to the manufacturer's protocol. The positive signals were stained with black CoS. Cells were counterstained with nuclear fast red at room temperature for $5 \mathrm{~min}$ to label the nucleus and cytoplasm of cells. Images were obtained using an inverted microscope (Nikon Eclipse TC 100; Nikon Corporation, Tokyo, Japan) at x100 magnification.

Determination of mineralization using von Kossa staining. Von Kossa staining (Sigma-Aldrich; Merck KGaA, Darmstadt, Germany) was used to determine the extent of minerals deposited on osteoblast cells. Silver nitrate solution $(0.5 \%)$ was added onto the cells and the plate was exposed to ultraviolet light for $30 \mathrm{~min}$. Subsequently, the cells were washed with saline and the reaction was stopped with the addition of $500 \mu 15 \%$ sodium thiosulfate (Sigma-Aldrich; Merck KGaA). Positive mineral depositions were stained in black. Cells were counterstained with nuclear fast red at room temperature for $5 \mathrm{~min}$ to label the nucleus and cytoplasm of cells. Images were obtained using an inverted microscope (Nikon Eclipse TC 100) at x100 magnification.

Western blot analysis. Immunoblotting was performed to detect the expression of Ihh, PTHrP, TGF- $\beta$, OCN, RUNX2, OPG, Smad2, Smad3, COL10A and RANKL in chondrocyte cells. Cultured or transfected cells were lysed in radioimmunoprecipitation assay buffer (Boster Biological Technology, Pleasanton, CA, USA) with $1 \%$ phenylmethylsulfonyl fluoride. The concentration of protein was determined using a BCA Protein Assay kit (cat. no. P0011, Beyotime Institute of Biotechnology) 

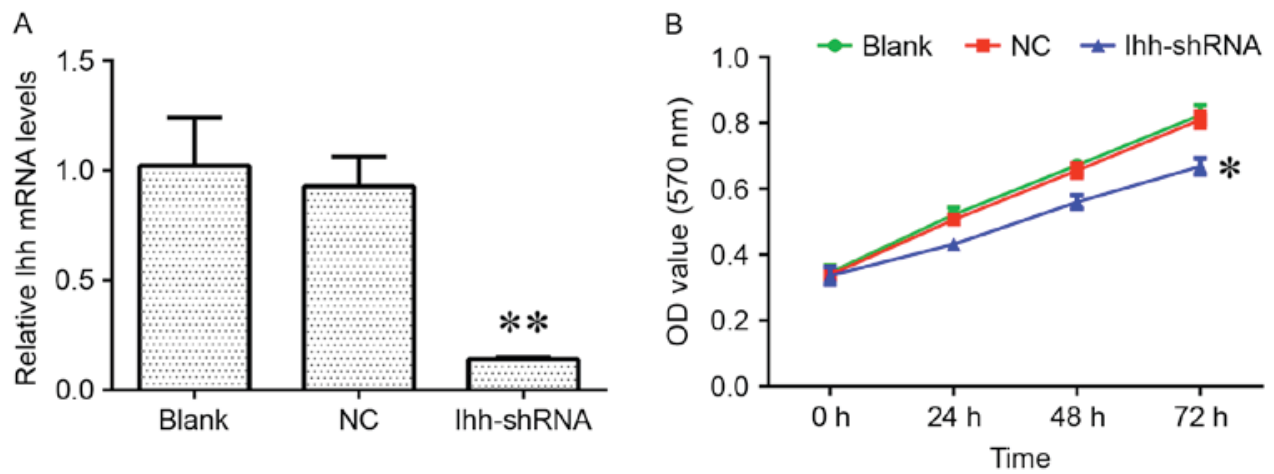

Figure 1. Ihh knockdown by shRNA inhibits MC3T3-E1 cells proliferation. (A) Reverse transcription-quantitative polymerase chain reaction analysis was used for detecting the expression of Ihh in MC3T3-E1 cells following transfection with Ihh shRNA lentivirus or empty lentivirus. (B) A Cell Counting Kit-8 assay was used for determining cell viability in MC3T3-E1 cells following transfection with Ihh shRNA lentivirus or empty lentivirus. ${ }^{*} \mathrm{P}<0.05,{ }^{* *} \mathrm{P}<0.01 \mathrm{vs}$. NC. Ihh, Indian hedgehog protein; shRNA, short hairpin RNA; OD, optical density; NC, negative control.

according to the manufacturer's protocol. Protein $(60 \mu \mathrm{g})$ was loaded onto a $12 \%$ SDS-PAGE minigel and transferred onto a polyvinylidene fluoride membrane. The membranes were blocked in $10 \%$ non-fat milk for $2 \mathrm{~h}$ at $37^{\circ} \mathrm{C}$. Following probing with primary antibodies (anti-Ihh, cat. no. SAB1403965, dilution, 1:1,000; anti-PTHrP, cat. no. SAB5300029, dilution, 1:1,000; anti-TGF- $\beta$, cat. no. SAB4502958, dilution, 1:2,000 and anti-OCN, cat. no. SAB1306277, dilution, 1:2,000 from Sigma Aldrich; Merck KGaA; anti-RUNX2, cat. no. 12556, dilution, 1:1,000; anti-OPG, cat. no. 4816, dilution, 1:1,000; anti-Smad2, cat. no. 5339, dilution, 1:1,000 and anti-Smad3, cat. no. 9523, dilution, 1:1,000 from Cell Signaling Technology, Inc., Danvers, MA, USA; anti-COL10A, cat. no. ab58632, dilution, 1:1,000; anti-RANKL, cat. no. ab9957, dilution, 1:1,000 and anti-GAPDH, cat. no. ab9485, dilution, 1:3,000 from Abcam, Cambridge, UK) at $4^{\circ} \mathrm{C}$ overnight, the blots were subsequently incubated with horseradish peroxidase-conjugated secondary antibody (cat. nos. ab7090 and ab97040; 1:5,000, Abcam) for $1 \mathrm{~h}$ at $37^{\circ} \mathrm{C}$. Signals were visualized using enhanced chemiluminescence substrates (EMD Millipore, Billerica, MA, USA). GAPDH was used as an endogenous protein for normalization. The densitometry of bands was analyzed using Quantity One software (version 4.6.9; Bio-Rad Laboratories, Inc., Hercules, CA, USA).

Statistical analysis. All data from three independent experiments are expressed as the mean \pm standard deviation and were processed using SPSS 17.0 statistical software (SPSS, Inc., Chicago, IL, USA). The difference among the groups was determined using Student's t-test or one-way analysis of variance with Tukey's post hoc test, depending on the conditions. $\mathrm{P}<0.05$ was considered to indicate a statistically significant difference.

\section{Results}

Knockdown of Ihh inhibits osteoblast cell proliferation, induces apoptosis and arrests the cell cycle at the G1 to $S$ phase. In order to investigate the biological role of Ihh in osteoblast cell growth, osteoblast MC3T3-E1 cells transfected with lentivirus containing Ihh shRNA were used for further analysis (Fig. 1A). The results of the present study demonstrated that the decreased expression of Ihh induced a significant inhibition of cell growth and markedly increased the apoptosis rate, compared with the $\mathrm{NC}$ group (Figs. 1B and 2A). In addition, the knockdown of Ihh significantly decreased the percentage of cells in the $\mathrm{S}$ phase, while increasing the percentage in the G1 phase, indicating a cell cycle arrest at G1 to S phase (Fig. 2B).

Knockdown of Ihh suppresses osteoblast cell differentiation. ALP is an enzyme which serves a role in bone mineralization. ALP activity was determined in the present study. Compared with the NC group, the ALP activity was decreased in chondrocytes with knocked-down Ihh (Fig. 3A). Calcification and mineralization of the bone matrix is essential for the strength and rigidity of the spinal skeletal system. In order to estimate the degree of mineralization and calcification, von Kossa staining following Ihh knockdown was performed. Representative images of von Kossa staining were obtained by brightfield microscopy, as presented in Fig. 3B. Compared with the NC group, the mineral deposition of osteoblasts lacking Ihh was decreased.

Effects of Ihh on TGF- $\beta /$ Smad and OPG/RANKL signaling. The present study further determined the effects of Ihh on TGF- $\beta /$ Smad and OPG/RANKL signaling, which is associated with bone differentiation and generation. As exhibited in Fig. 4, the mRNA expression of COL10A, RANKL, Smad2 and Smad3 was significantly upregulated, although the levels of OCN, OPG, PTHrP, RUNX2 and TGF- $\beta$ were significantly decreased, following knockdown of Ihh in osteoblast cells. The protein expression of these genes was analyzed using western blotting. As presented in Fig. 5, the protein expression of COL10A, RANKL, Smad2 and Smad3 was significantly upregulated, while the levels of OCN, OPG, PTHrP, RUNX2 and TGF- $\beta$ were significantly decreased, following knockdown of Ihh in osteoblast cells. The results of the present study suggested that the inhibitory effects of Ihh knockdown on bone differentiation and generation are associated with the TGF- $\beta /$ Smad and OPG/RANKL signaling pathways.

\section{Discussion}

In the present study, it was observed that the knockdown of Ihh induced a significant inhibition of cell growth and markedly 

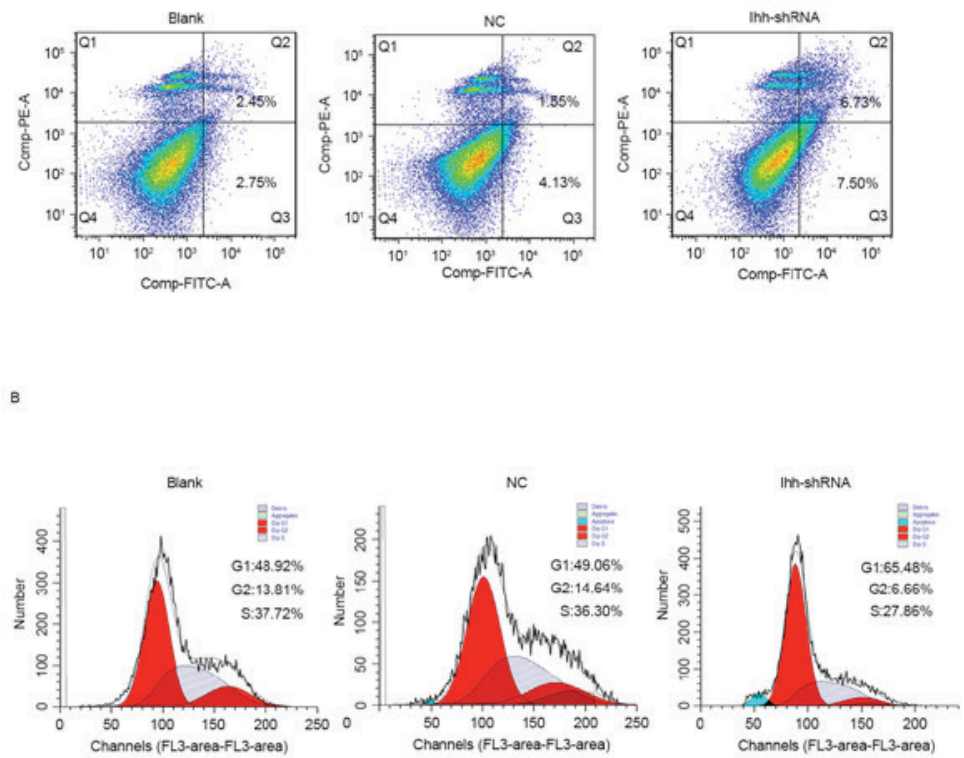
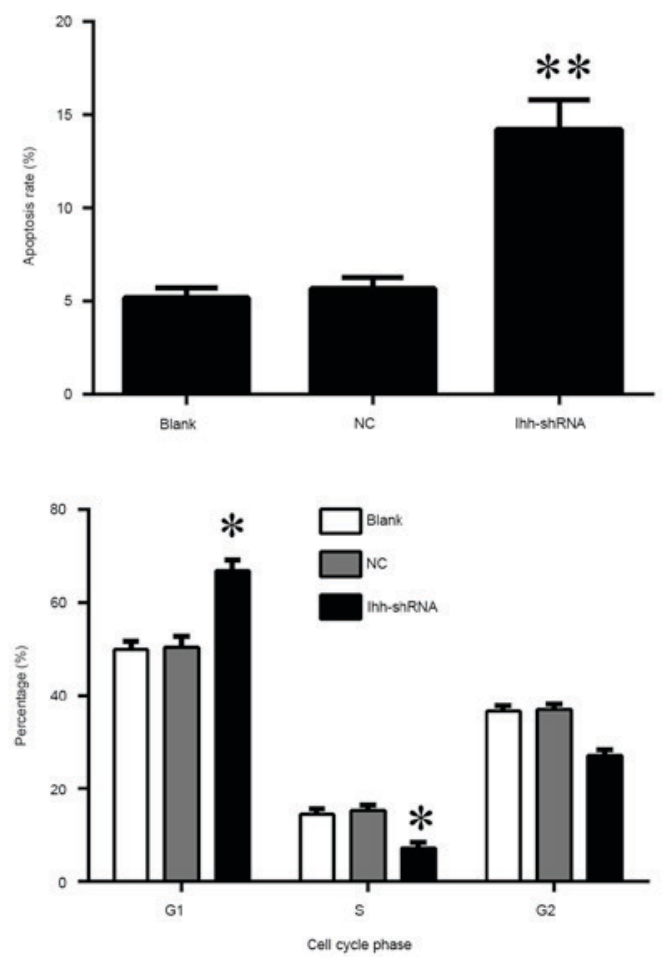

Figure 2. Effects of Ihh knockdown on cell apoptosis and cell cycle. (A) The apoptosis rate was measured using flow cytometry in MC3T3-E1 cells, following transfection with Ihh shRNA lentivirus or empty lentivirus. (B) Cell cycle distribution was measured by flow cytometry in MC3T3-E1 cells, following transfection with Ihh shRNA lentivirus or empty lentivirus. ${ }^{*} \mathrm{P}<0.05,{ }^{* *} \mathrm{P}<0.01$ vs. NC control. Ihh, Indian hedgehog protein; shRNA, short hairpin RNA; $\mathrm{NC}$, negative control.

A

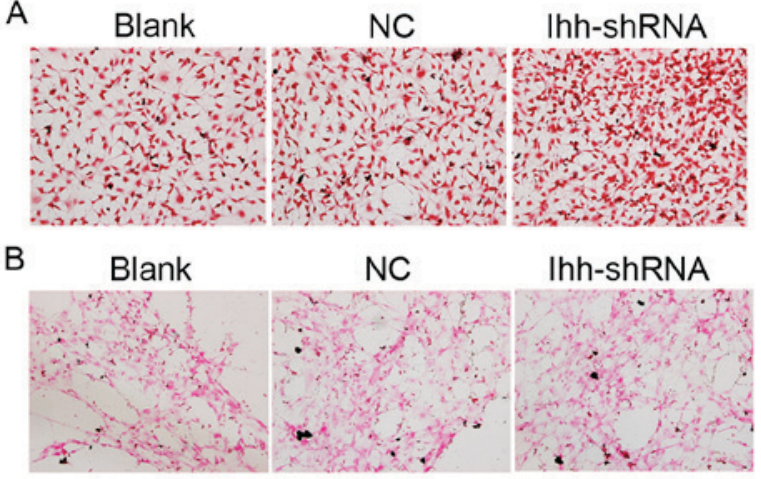

Figure 3. Effects of Ihh knockdown on alkaline phosphatase activity and mineral deposition in MC3T3-E1 cells. (A) Alkaline phosphatase activity was decreased in MC3T3-E1 cells with knocked-down Ihh compared with NC. Magnification, x100. (B) Von Kossa staining was used to determine the extent of mineral deposition in MC3T3-E1 cells. Compared with NC, the mineral deposition of MC3T3-E1 cells with knocked-down Ihh was decreased. Magnification, x100. Ihh, Indian hedgehog protein; shRNA, short hairpin RNA; NC, negative control.

increased the apoptosis rate, compared with the NC group, in osteoblasts. Downregulation of Ihh resulted in a cell cycle arrest at the G1 to S phase in osteoblasts. In addition, knockdown of Ihh decreased the ALP activity and mineral deposition in osteoblasts. The inhibitory roles of Ihh downregulation in osteoblast growth and differentiation may be associated with the TGF- $\beta /$ Smad and OPG/RANKL signaling pathways.

Various hormones and factors, including Hh proteins and transcription factors, regulate osteoblast differentiation. Ihh is an essential transcription factor for osteoblast differentiation

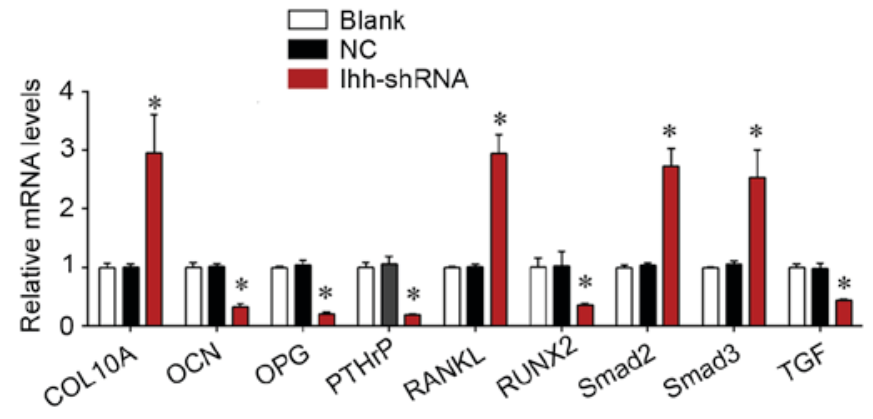

Figure 4. Reverse transcription-quantitative polymerase chain reaction analysis was used for detecting the mRNA expression of COL10A, RANKL, Smad2, Smad3, OCN, OPG, PTHrP, RUNX2 and TGF- $\beta$ in MC3T3-E1 cells following transfection with Ihh shRNA lentivirus or empty lentivirus. ${ }^{*} \mathrm{P}<0.05$ vs. NC. COL10A, collagen $\alpha-1$ (X) chain; RANKL, tumor necrosis factor ligand superfamily member 11 ; Smad, mothers against decapentaplegic homolog; OCN, osteocalcin; OPG, tumor necrosis factor receptor superfamily member 11B; PTHrP, parathyroid hormone-related protein; RUNX2, runt-related transcription factor 2 ; TGF- $\beta$, transforming growth factor- $\beta$; Ihh, Indian hedgehog protein; shRNA, short hairpin RNA; NC, negative control.

and bone formation, which acts by interacting with bone morphogenetic proteins (11). Osteoblast MC3T3-E1 cells expressing Ihh exhibited increased ALP activity, which was synergistically enhanced by bone morphogenetic protein-2, indicating that $\mathrm{Hh}$ proteins directly act on osteogenic precursor cells and osteoblasts to stimulate osteogenic differentiation (12). The removal of smoothened homolog, which triggers Hh signaling, from perichondrial cells prevents the formation of a normal bone collar and abolishes the development of the primary spongiosa $(13,14)$. Ihh knockdown results in markedly 

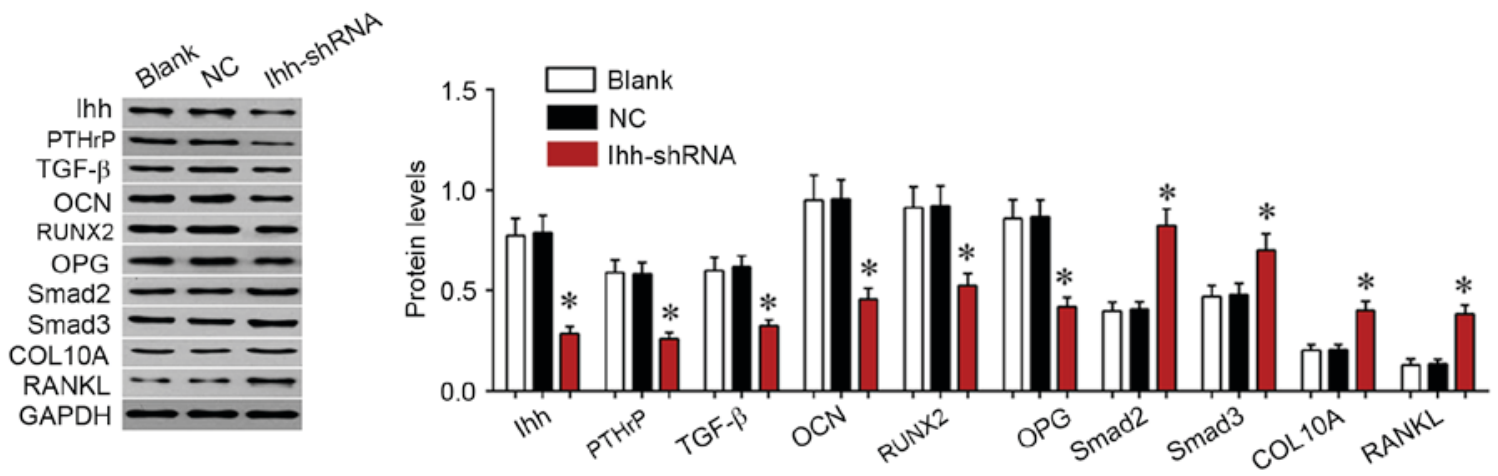

Figure 5. Western blot analysis of the expression of COL10A, RANKL, Smad2, Smad3, OCN, OPG, PTHrP, RUNX2 and TGF- $\beta$ in MC3T3-E1 cells following transfection with Ihh shRNA lentivirus or empty lentivirus. "P<0.05 vs. NC. COL10A, collagen $\alpha-1$ (X) chain; RANKL, tumor necrosis factor ligand superfamily member 11; Smad, mothers against decapentaplegic homolog; OCN, osteocalcin; OPG, tumor necrosis factor receptor superfamily member 11B; PTHrP, parathyroid hormone-related protein; RUNX2, runt-related transcription factor 2 ; TGF- $\beta$, transforming growth factor- $\beta$; Ihh, Indian hedgehog protein; shRNA, short hairpin RNA; NC, negative control

foreshortened limbs and a complete absence of mature osteoblasts, and acts as an important positive regulator of cranial bone ossification (15). Ectopic Ihh promotes the progression of the bone collar towards the epiphysis, and Hh signaling is found to be required in BMP-induced osteogenesis in cultures of a limb-bud cell line, suggesting that Ihh signaling is directly required for the osteoblast lineage in the developing long bones and that Ihh functions in conjunction with other factors such as BMPs to induce osteoblast differentiation (14). In addition, Hh proteins promote osteoblast recruitment and differentiation, possibly by stimulating PTHrP expression and secretion (16). RUNX2 is a transcription factor which is required for osteoblast differentiation. RUNX2 overexpression has been demonstrated to stimulate chondrocyte maturation, as indicated by increases in ALP activity, mineralization, and COL10A and matrix metalloproteinase-13 expression, and by maintenance of Ihh expression, whereas PTHrP treatment blocked RUNX2 expression and its promaturation effects (17). However, forced expression of RUNX2 in skeletogenic cells restores bone formation in the Runx2-null, although not in the Ihh-null, embryo, indicating that in addition to Runx2, Ihh requires other effectors to induce osteoblast differentiation, including zinc finger proteins Gli2 and Gli3 $(8,18,19)$. Wnt signaling acts downstream of Ihh signaling and is required in osteoblast maturation during endochondral bone formation; to enhance fracture repair or bone formation, Ihh signaling requires enhancement at the early stages by controlling osteoblast proliferation and differentiation, whereas Wnt signaling may be upregulated later in differentiated osteoblasts $(1,20)$. Notably, osteoblast differentiation and bone formation may occur in conditions of specific ablation of Ihh in the limb mesenchyme $(2,21)$.

In the present study, it was observed that the mRNA and protein expression of osteoblastic and osteocytic markers, including COL10A, RANKL, Smad2 and Smad3 was notably upregulated, while the levels of OCN, OPG, PTHrP, RUNX2 and TGF- $\beta$ were significantly decreased following knockdown of Ihh in osteoblasts. TGF- $\beta$ signaling exerts important roles in embryonic skeletal development and postnatal bone homeostasis, by transducing signals in the canonical Smad-dependent signaling pathway to regulate mesenchymal stem cell differentiation during skeletal development, bone formation and bone homeostasis (22). The coordinated activity of RUNX2 and TGF- $\beta$-activated Smad proteins is important for the formation of the skeleton (23). Knockout or mutation of TGF- $\beta$ signaling-associated genes in mice leads to bone abnormalities of varying severity (24). For example, $\mathrm{AlCl} 3$ has been demonstrated to inhibit osteoblast mineralization via the TGF- $\beta 1 /$ Smad signaling pathway in rat osteoblasts (25).

In conclusion, the knockdown of Ihh suppressed osteoblast growth and differentiation via a mechanism which may be associated with the TGF- $\beta /$ Smad and OPG/RANKL signaling pathways. The results of the present study suggested that osteoblast-derived Ihh is essential for maintaining the growth plate. The manipulation of Ihh expression or its signaling components may be of benefit in the treatment of skeletal diseases.

\section{Acknowledgements}

The present study was supported by the Natural Science Foundation of Hunan Province, China (grant no. 2016JJ3160) and the National Natural Science Foundation of China (grant no. 81472145).

\section{References}

1. Day TF and Yang Y: Wnt and hedgehog signaling pathways in bone development. J Bone Joint Surg Am 90 (Suppl 1): S19-S24, 2008.

2. Yang J, Andre P, Ye L and Yang YZ: The Hedgehog signalling pathway in bone formation. Int J Oral Sci 7: 73-79, 2015.

3. Xing W, Cheng S, Wergedal J and Mohan S: Epiphyseal chondrocyte secondary ossification centers require thyroid hormone activation of Indian hedgehog and osterix signaling. J Bone Miner Res 29: 2262-2275, 2014.

4. Komori T: Signaling networks in RUNX2-dependent bone development. J Cell Biochem 112: 750-755, 2011.

5. Hart GT, Burton EL and Mincer HH: Use of screening blood studies in dental schools. J Dent Educ 55: 735-737, 1991.

6. Kronenberg HM and Chung U: The parathyroid hormone-related protein and Indian hedgehog feedback loop in the growth plate. Novartis Found Symp 232: 144-157, 2001.

7. Colnot C, de la Fuente L, Huang S, Hu D, Lu C, St-Jacques B and Helms JA: Indian hedgehog synchronizes skeletal angiogenesis and perichondrial maturation with cartilage development. Development 132: 1057-1067, 2005. 
8. Shimoyama A, Wada M, Ikeda F, Hata K, Matsubara T, Nifuji A, Noda M, Amano K, Yamaguchi A, Nishimura R and Yoneda T: Ihh/Gli2 signaling promotes osteoblast differentiation by regulating Runx2 expression and function. Mol Biol Cell 18: 2411-2418, 2007.

9. Cai JQ, Huang YZ, Chen XH, Xie HL,Zhu HM, Tang L, Yang ZM, Huang YC and Deng L: Sonic hedgehog enhances the proliferation and osteogenic differentiation of bone marrow-derived mesenchymal stem cells. Cell Biol Int 36: 349-355, 2012.

10. Livak KJ and Schmittgen TD: Analysis of relative gene expression data using real-time quantitative PCR and the 2(-Delta Delta C(T)) method. Methods 25: 402-408, 2001.

11. Yamaguchi A, Komori T and Suda T: Regulation of osteoblast differentiation mediated by bone morphogenetic proteins, hedgehogs, and Cbfa1. Endocr Rev 21: 393-411, 2000.

12. Nakamura T, Aikawa T, Iwamoto-Enomoto M, Iwamoto M, Higuchi Y, Pacifici M, Kinto N, Yamaguchi A, Noji S, Kurisu K and Matsuya T: Induction of osteogenic differentiation by hedgehog proteins. Biochem Biophys Res Commun 237 465-469, 1997

13. Pacheco M, Valencia M, Caparros-Martin JA, Mulero F, Goodship JA and Ruiz-Perez VL: Evc works in chondrocytes and osteoblasts to regulate multiple aspects of growth plate development in the appendicular skeleton and cranial base. Bone 50 : 28-41, 2012.

14. Long F, Chung UI, Ohba S, McMahon J, Kronenberg HM and McMahon AP: Ihh signaling is directly required for the osteoblast lineage in the endochondral skeleton. Development 131: 1309-1318, 2004.

15. Lenton K, James AW, Manu A, Brugmann SA, Birker D, Nelson ER, Leucht P, Helms JA and Longaker MT: Indian hedgehog positively regulates calvarial ossification and modulates bone morphogenetic protein signaling. Genesis 49: 784-796, 2011.

16. Jemtland R, Divieti P, Lee K and Segre GV: Hedgehog promotes primary osteoblast differentiation and increases PTHrP mRNA expression and iPTHrP secretion. Bone 32: 611-620, 2003.
17. Iwamoto M, Kitagaki J, Tamamura Y, Gentili C, Koyama E, Enomoto H, Komori T, Pacifici M and Enomoto-Iwamoto M: Runx2 expression and action in chondrocytes are regulated by retinoid signaling and parathyroid hormone-related peptide (PTHrP). Osteoarthritis Cartilage 11: 6-15, 2003.

18. Tu X, Joeng KS and Long F: Indian hedgehog requires additional effectors besides Runx2 to induce osteoblast differentiation. Dev Biol 362: 76-82, 2012

19. Hilton MJ, Tu X, Cook J, Hu H and Long F: Ihh controls cartilage development by antagonizing Gli3, but requires additional effectors to regulate osteoblast and vascular development. Development 132: 4339-4351, 2005.

20. Maeda Y, Nakamura E, Nguyen MT, Suva LJ, Swain FL, Razzaque MS, Mackem S and Lanske B: Indian Hedgehog produced by postnatal chondrocytes is essential for maintaining a growth plate and trabecular bone. Proc Natl Acad Sci USA 104: 6382-6387, 2007

21. Amano K, Densmore MJ and Lanske B: Conditional deletion of Indian hedgehog in limb mesenchyme results in complete loss of growth plate formation but allows mature osteoblast differentiation. J Bone Miner Res 30: 2262-2272, 2015.

22. Hendy GN, Kaji H, Sowa H, Lebrun JJ and Canaff L: Menin and TGF-beta superfamily member signaling via the Smad pathway in pituitary, parathyroid and osteoblast. Horm Metab Res 37: 375-379, 2005.

23. Chen G, Deng C and Li YP: TGF- $\beta$ and BMP signaling in osteoblast differentiation and bone formation. Int J Biol Sci 8: 272-288, 2012

24. Wu M, Chen G and Li YP: TGF- $\beta$ and BMP signaling in osteoblast, skeletal development, and bone formation, homeostasis and disease. Bone Res 4: 16009, 2016.

25. Sun X, Cao Z, Zhang Q, Li M, Han L and Li Y: Aluminum trichloride inhibits osteoblast mineralization via TGF- $\beta 1 / \mathrm{Smad}$ signaling pathway. Chem Biol Interact 244: 9-15, 2016. 